\title{
Prelimbic Cortical Neurons Track Preferred Reward Value and Reflect Impulsive Choice during Delay Discounting Behavior
}

\author{
D. Deirdre A. Sackett, Travis M. Moschak, and Regina M. Carelli \\ Department of Psychology and Neuroscience, The University of North Carolina, Chapel Hill, North Carolina 27599
}

In delay discounting, individuals discount the value of a reward based on the delay to its receipt. The prelimbic cortex (PrL) is heavily interconnected with several brain regions implicated in delay discounting, but the specific contributions of the PrL to delay discounting are unknown. Here, we used multineuron electrophysiological recording methods in Long-Evans male $(n=10)$ and female $(n=9)$ rats to characterize the firing dynamics of PrL neurons during discrete cue and lever press events in a delay discounting task. Rats' initial preference for the large reward decreased as delays for that outcome increased across blocks, reflecting classic discounting behavior. Electrophysiological recordings revealed that subgroups of neurons exhibited phasic responses to cue presentations and lever presses. These phasic neurons were found to respond to either large/delay, small/immediate, or both trial types and the percentage of these neurons shifted across blocks as the expected value of the reward changed. Critically, this shift was only seen during trials in which animals could choose their preferred option (free choice trials) and not during trials where animals could choose only one option (forced choice trials). Further, this shift was dependent on rats' inherent impulsivity because high impulsive rats demonstrated a greater percentage of small/immediate-responsive neurons as the task progressed. Collectively, these findings suggest a unique role for the PrL in encoding reward value during delay discounting that is influenced by individual differences in impulsivity.

Key words: accumbens; behavior; decision making; impulsivity; prefrontal; prelimbic

Significance Statement

In delay discounting, individuals discount the value of a reward based on the delay to its receipt. Here, we used electrophysiology to investigate the role of the prelimbic cortex (PrL) in this process. We found that subsets of neurons shifted activity as a function of the changing expected delay and reward magnitude, but this shift was only evident during trials in which animals could choose their preferred option. Further, this dynamic neural activity depended on rats' inherent impulsivity, with impulsive rats exhibiting a stronger neural shift toward the immediate reward as the task progressed. These findings suggest a role for the PrL in encoding reward value during delay discounting that is influenced by goal-directed context and individual differences in impulsivity.

\section{Introduction}

Delay discounting is a decision-making process in which the subjective value of a reward decreases as the delay to its receipt increases (Roesch et al., 2006, 2007; Roesch and Bryden, 2011; Tedford et al., 2015). When the delay becomes too long, individuals will shift their preference from a large delayed reward to a smaller, immediate one. In this way, delay discounting serves as

Received 0ct. 1, 2018; revised Jan. 28, 2019; accepted Feb. 3, 2019.

Author contributions: D.A.S. performed research; D.A.S. and T.M.M. analyzed data; D.A.S. wrote the first draft of the paper; T.M.M. and R.M.C. edited the paper; R.M.C. designed research; R.M.C. wrote the paper.

This work was supported by the National Institutes of Health (Grant DA034021 to R.M.C. and Grants T32 DA007244 and F31 DA042721-01 to D.A.S.). We thank Xuefei Wang for outstanding technical support.

The authors declare no competing financial interests.

Correspondence should be addressed to Regina M. Carelli at rcarelli@unc.edu.

https://doi.org/10.1523/JNEUROSCI.2532-18.2019

Copyright $@ 2019$ the authors an index of impulsivity because it measures an individual's unwillingness to delay gratification for a larger reward. Importantly, heightened delay discounting (increased impulsivity) is a common symptom of numerous psychiatric disorders (Jentsch and Taylor, 1999; Barkley et al., 2001; Crews and Boettiger, 2009). Therefore, understanding the neural circuitry mediating delay discounting is a timely and important topic.

Recent studies have begun to elucidate the neural circuitry underlying delay discounting. For example, in the nucleus accumbens (NAc) core, dopaminergic activity tracks the value of the predicted reward in a delay discounting task (Saddoris et al., 2015; Moschak and Carelli, 2017). Additionally, lesions to the NAc core promote impulsive choice during delay discounting (Cardinal et al., 2001), although 6-OHDA lesions to the NAc core do not (Winstanley et al., 2005b). Furthermore, individual dif- 
ferences in delay discounting predict individual variations in dopamine activity (Diergaarde et al., 2008; Moschak and Carelli, 2017; but see Zeeb et al., 2016). Additionally, rewards of differing delays and magnitudes are tracked by neural activity in the NAc, basolateral amygdala (BLA), orbitofrontal cortex, and ventral tegmental area (VTA) (Roesch et al., 2006, 2007, 2009, 2010) and inactivation or lesions to the orbitofrontal cortex and BLA disrupt delay discounting behavior (Mobini et al., 2002; Winstanley et al., 2004; Zeeb et al., 2010).

However, one area that has received relatively little attention with respect to delay discounting is the prelimbic cortex (PrL). Recent studies indicate that the rodent PrL is the homolog of the human anterior cingulate cortex (ACC) (Heilbronner et al., 2016) and is heavily interconnected with other brain regions implicated in delay discounting, including the NAc core and BLA. Although several studies have implicated the human ACC in delay discounting (McClure et al., 2004; Monterosso et al., 2007; Peters and Büchel, 2010), preclinical studies have yielded mixed results. Some studies have reported that inactivation or lesion of the medial PFC, which includes the PrL area, increases delay discounting (Churchwell et al., 2009; Gill et al., 2010), whereas others have found no effect (Cardinal et al., 2001; Feja and Koch, 2014). Additional work has suggested that individual differences in impulsivity may be reflected in PrL function because high PrL D2 mRNA expression predicted a higher preference for large, delayed rewards (Simon et al., 2013). However, it remains unknown how neurons in the PrL specifically encode information related to delay discounting and impulsive choice.

Here, we used electrophysiological recording in male and female rats to examine PrL activity during a delay discounting task that varied subjective costs (delay to reward) across the session. Rats could choose between a small reward (1 sugar pellet) available immediately versus a large reward ( 3 sugar pellets) available after no delay (0 s), a short delay (10 s), or a long delay (20 s). Neuronal activity was examined during cues that predicted the availability of either the small/immediate or large/delay reward, as well as relative to the response (lever press) for reward. We found that subsets of PrL neurons tracked discrete cue presentations and in some cases exhibited activity that was responsive to either large/delay or small/immediate trial types, but only during trials in which rats could choose their preferred outcome. Further, these tracking dynamics differed based on level of impulsivity, with high impulsive (HI) rats exhibiting more small/ immediate-responsive neurons as the task progressed. Together, the findings indicate a unique role of PrL neurons in tracking preferred reward outcome during delay discounting and impulsive choice.

\section{Materials and Methods}

Subjects. Singly housed male $(n=10)$ and female $(n=9)$ Long-Evans rats ( $\sim 90$ to $120 \mathrm{~d}$ old) weighing $275-330 \mathrm{~g}$ at the start of experiments were used. Animals were maintained at no less than $85 \%$ of preexperimental body weights by food restriction except during the postoperative recovery period, when food (Purina Lab Chow) was given ad libitum. Water was available ad libitum. Animal procedures were conducted in accordance with the National Institutes of Health's Guidelines for the Care and Use of Laboratory Animals and were approved by the University of North Carolina at Chapel Hill Institutional Animal Care and Use Committee.

Apparatus. Behavioral testing was conducted in $43 \times 43 \times 53 \mathrm{~cm}$ Plexiglas chambers housed in sound-blocking boxes (Med Associates) described in detail previously (Saddoris et al., 2011). Briefly, one side of each chamber was equipped with two retractable levers (Coulbourn Instruments) $17 \mathrm{~cm}$ apart with a stimulus light $6 \mathrm{~cm}$ above each lever.
Sucrose pellets ( $45 \mathrm{mg}$ for males, $20 \mathrm{mg}$ for females) were delivered to a food receptacle, which was located equidistantly between the levers. A house light $(100 \mathrm{~mA})$ was mounted on the opposite side of the chamber.

Surgery. Rats were deeply anesthetized with a ketamine hydrochloride $(100 \mathrm{mg} / \mathrm{kg})$ and xylazine hydrochloride $(10 \mathrm{mg} / \mathrm{kg})$ mixture intraperitoneally and given an anti-inflammatory medication (meloxicam, $1 \mathrm{mg} /$ $\mathrm{kg}$, s.c.) before surgery and for $2 \mathrm{~d}$ after surgery. Microwire electrode arrays consisting of 8 microwires (50 $\mu \mathrm{m}$ in diameter, NB Labs), described previously in Carelli et al. (2000), were bilaterally implanted in the prelimbic cortex (AP +2.7, \pm ML $0.6, \mathrm{DV}-4.0$ from bregma) and secured in place with dental cement and stainless steel screws. Following surgery, rats were allowed to recover for 1 week with ad libitum food and water.

Behavioral procedures. All behavioral experiments were conducted at least 1 week after surgery. Rats were initially trained to press two distinct levers in which each response was reinforced on a continuous schedule of reinforcement. Reinforced responses resulted in the delivery of a sucrose pellet to a centrally located food cup. Animals were trained to a criterion of 50 presses on each response lever.

Next, rats were trained on a delay discounting task used previously (Saddoris et al., 2015) and based on a paradigm designed by Evenden and Ryan (1996). The task was composed of three trial types. On forced choice delay trials (Fig. 1A, left), a cue light was illuminated for $5 \mathrm{~s}$, followed by extension of two levers. A single press on the lever positioned below the illuminated cue light resulted in a large reward (three sucrose pellets) delivered after a period of delay, as described below. During forced choice immediate trials (Fig. 1A, middle), another $5 \mathrm{~s}$ cue light signaled that responses on the associated lever resulted in a small (one sucrose pellet) immediate reward. On free choice trials (Fig. 1A, right), both cue lights illuminated for $5 \mathrm{~s}$, signaling that both responses were rewarded based on the contingency of the lever chosen. Each behavioral session consisted of three blocks of trials: during block 1, the large reward was presented immediately (no-delay block); in block 2 , the delay to large reward was $10 \mathrm{~s}$ following a lever press (short-delay block); in block 3 , there was a $20 \mathrm{~s}$ delay to obtain the large reward (long-delay block). Rats performed 30 trials per block ( 20 forced choice; 10 of each type, and 10 free choice trials). The forced choice delay and immediate trials were intermingled and presented before free choice trials. If animals failed to respond within $10 \mathrm{~s}$, then both levers retracted and the trial was counted as an omission. Because each trial was a fixed duration (60 s), reward choice did not influence how quickly the rat completed the task (i.e., choosing the small reward did not lead to the next trial quicker). Once trained on the task (4-6 weeks), rats underwent electrophysiological recording to determine PrL neuronal activity during discrete task events.

Electrophysiological recordings. Electrophysiological procedures have been described in detail previously (Day et al., 2011; West et al., 2014; Moschak et al., 2018). Briefly, before the start of each session, the subject was connected to a flexible recording cable attached to a commutator (Med Associates), which allowed virtually unrestrained movement within the chamber. The head stage of each recording cable contained 16 miniature unity-gain field effect transistors. Neurons were recorded differentially between each active and the inactive (reference) electrode from the permanently implanted microwires. The inactive electrode was examined before the start of the session to verify the absence of neuronal spike activity and served as the differential electrode for other electrodes with cell activity. Online isolation and discrimination of neuronal activity was accomplished using a commercially available neurophysiological system, the multichannel acquisition processor (MAP) system (Plexon). Multiple window discrimination modules and high-speed analog-todigital signal processing in conjunction with computer software enabled isolation of neuronal signals based on waveform analysis. The neurophysiological system incorporated an array of digital signal processors (DSPs) for continuous spike recognition. The DSPs provided a continuous parallel digital output of neuronal spike events to a Pentium computer. Another computer processed operant chamber input and output (Med Associates) and sent digital outputs corresponding to each event to the MAP box to be time stamped along with the neural data. Discrimination of individual waveforms began by setting a threshold level (well above background noise) for each wire. Units detected had to display 
peak voltage at least $20 \%$ greater than baseline. Individual waveforms corresponding to a single cell were discriminated using template analysis procedures and time-voltage boxes provided by the MAP system. Cell recognition and sorting were finalized after the experiment using the Offline Sorter program (Plexon). This allowed neuronal data to be further assessed based on the principle component analysis of the waveforms, cell firing characteristics such as autocorrelograms and interspike interval distributions to ensure that putative cells showed biologically appropriate firing refractory periods, and cross-correlograms to ensure that multiple cells recorded on the same wires showed firing independently of each other. Waveform and spontaneous firing rates were examined to identify putative glutamatergic pyramidal neurons in the PrL and exclude GABAergic interneurons from analysis (Moorman and Aston-Jones, 2015; Devilbiss et al., 2017).

Classification of $\operatorname{PrL}$ neurons relative to task events. Changes in neuronal firing patterns relative to each behavioral event were analyzed by constructing perievent histograms and raster displays (bin width, $250 \mathrm{~ms}$ ) surrounding each task event (cue, lever press) using commercially available software (Neuroexplorer for Windows version 4.034; Plexon). Each cell was examined for changes in activity relative to cue onset ( 0 to $5 \mathrm{~s}$ following cue presentation) and/or following the lever press ( 0 to $2.5 \mathrm{~s}$ after response completion). Individual units were categorized as showing either a decrease (inhibition) or an increase (excitation) in firing rate compared with baseline (i.e., termed "phasic") or no difference from baseline (termed "nonphasic"). Specifically, cells were classified as phasic if during one of these epochs the firing rate was greater than or less than the $95 \%$ confidence interval (CI) projected from the baseline period ( -10 to $0 \mathrm{~s}$ before cue onset or -10 to $-2.5 \mathrm{~s}$ before a lever press) for at least one $250 \mathrm{~ms}$ time bin. This confidence interval was selected such that only robust responses were categorized as excitatory or inhibitory following established procedures (Day et al., 2011; West et al., 2014). Some neurons in this analysis exhibited low baseline firing rates, and the $95 \%$ $\mathrm{CI}$ included zero. Where this was the case, inhibitions were assigned if $e_{0}$ $>2^{\star} b_{0}$ (where $e_{0}=$ the number of consecutive 0 spikes/s time bins during the event epoch and $b_{0}=$ the maximal number of consecutive 0 spikes/s time bins during the baseline period). Units that exhibited both excitations and inhibitions within the same epoch were classified by the response that was most proximal to the event in question unless the most proximal response was ongoing when the event occurred. Importantly, the above analysis was completed separately for forced choice large/delay and small/immediate trial types, as well as during free choice trials when rats eventually chose the large/delay or small/immediate reward. This allowed determination as to how many neurons responded to each cue and lever press event in each block and trial type. However, the resultant categories of neuronal response profiles were not mutually exclusive. For example, a neuron could potentially exhibit an excitation to the forced large/delay cue and an inhibition to the forced small/immediate or an excitation to both forced large/delay and small/immediate cues. Additionally, during some free choice trial blocks, some rats only chose one lever. Therefore, in these instances, neurons could not be classified as phasic or nonphasic for the unchosen lever and these neurons were not included in the analysis. Finally, we also compared neural firing rate during the task with methods used previously (Day et al., 2011). Firing rate for each cell was normalized with a $z$-score transformation using the average and SD from the baseline periods noted above. For each block, we then calculated the average normalized firing rate in the $5 \mathrm{~s}$ period following cue onset or lever press.

Classification of phasic neurons putatively responsive to task events. Phasic neurons were further characterized as "putatively responsive" to discrete task events (cue or press) during either forced or free choice trials. These putatively responsive phasic neurons were classified as one of three types. The first type was "putative large/delay responsive." These neurons were phasic (either excitatory or inhibitory) during either the cue or lever press during forced choice large/delay trials or when rats chose the large/ delay option during free choice and were nonphasic during small/immediate trials. The second type was "putative small/immediate responsive." These neurons were phasic during task events (cue, press) on forced choice small/immediate trials or when rats chose the small/immediate option during free choice trials and were nonphasic during large/delay trials. Finally, other neurons were responsive to both trial types. That is, these neurons were phasic during both large/delay and small/immediate trials regardless of rats' free choice selection. Importantly, free choice trials present both cue lights simultaneously for $5 \mathrm{~s}$, allowing rats to choose the preferred option, so we classified cue-responsive neurons based on rats' eventual choice. For example, when rats chose the large/ delay option, phasic neurons during the preceding cue period were labeled as putative "large/delay" responsive. Furthermore, because forced and free choice trials were analyzed separately, neuron populations were not mutually exclusive to forced or free trial types. For example, a neuron putatively responsive to forced choice large/delay trials could also be responsive to free choice large/delay trials.

Statistics. Before recording, stability in task performance was assessed for each rat by visually inspecting discounting curves for three sessions before recording. To confirm stability, we used ANOVA to analyze percentage delay choice across the three sessions before recording and found no significant difference across sessions. 
To determine whether sex differences in behavioral responding existed during the task, percentage delay choice chosen across blocks (discounting curve) and accuracy in the task were both analyzed using a two-way ANOVA (block $\times$ sex). Accuracy was defined as the percentage of forced choice trials in which the animal made the correct response (for each block). Because there were no differences in any measure between sexes, all rats were combined for further analyses.

Rats were separated into HI $(n=9)$ and low impulsive $(n=9, \mathrm{LI})$ groups using a median split on average discounting score (the average of delay choice across blocks). Percentage correct responses (accuracy) on forced choice trials were analyzed using a one-way ANOVA for HI and LI rats. Percentage delay choice chosen across blocks (discounting curve) was analyzed using a two-way ANOVA (block $\times$ impulsivity trait).

Differences in the frequency or proportion of neuronal responses across different trial types were examined using $\chi^{2}$ or Fisher's exact test. All analyses were considered significant at $\alpha=0.05$. Statistical and graphical analysis was conducted in GraphPad Prism 4 software.

Histology. Upon completion of the experiment, rats were deeply anesthetized with a ketamine and xylazine mixture $(100 \mathrm{mg} / \mathrm{kg}$ and $10 \mathrm{mg} / \mathrm{kg}$, i.p., respectively). To mark the placement of electrode tips, a $13.5 \mu \mathrm{A}$ current was passed through each microwire electrode for $5 \mathrm{~s}$. Brains were removed and placed into a formalin solution with $20 \%$ sucrose and $3 \%$ potassium ferricyanide, after which $40 \mu \mathrm{m}$ coronal brain sections were sliced and mounted on slides. The addition of potassium ferricyanide allowed for a blue reaction corresponding to the location of individual electrode tips, which was assessed by visual examination of successive coronal sections. Placement of an electrode tip within the PrL was determined by examining the relative position of observable reaction product to visual landmarks and anatomical organization of the rodent medial prefrontal cortex represented in a stereotaxic atlas (Paxinos and Watson, 1998).

\section{Results}

\section{Behavior}

Animals reliably acquired the delay discounting task and were stable (no change in delay discounting across 3 sessions, $F_{(2,34)}=$ $1.50, p=0.237)$ and there were no sex differences. Specifically, there was no significant difference in accuracy $\left(F_{(1,17)}=0.3102\right.$, $p=0.5848)$ or delay choice (discounting curve) $\left(F_{(1,17)}=0.1442\right.$, $p=0.7089$ ) between males and females (Fig. 1B). Therefore, for the remainder of this analysis, males and females were combined. All rats responded accurately to forced choice trials and made significantly more errors on block 3 forced choice trials $\left(F_{(2,36)}=\right.$ $4.499, p=0.0180$; Fig. 1C).

\section{Task-related neuronal activity}

A total of 125 individual PrL neurons were recorded from 19 animals ( $n=10$ male, $n=9$ female) during behavioral performance. Waveform analysis revealed that 115 neurons were putative pyramidal (i.e., glutamatergic) neurons; the remaining 10 were excluded from analysis. PrL neurons were classified as exhibiting phasic activity (increases and/or decreases in firing rate) relative to task cues and/or lever presses, as described below.

Task cues evoked changes in firing rate in a large population of PrL neurons. Of the 115 neurons, 96 cells (83.5\%) exhibited phasic changes in firing rate during at least one forced choice cue presentation and 88 (76.5\%) exhibited changes during at least one free choice cue presentation. These phasic neurons were classified as cue-excitation (Fig. 2A, left) or cue-inhibition (Fig. 2A, right). During forced choice cue presentations, when rats could only choose one reward option versus no reward, there was no significant difference between the percentage of phasic neurons across blocks for either large/delay trials or small/immediate trials ( $p>0.05$ for all analyses; data not shown). However, during the free choice cue presentations (both cue lights were presented) that preceded the choice for the large/delay option, PrL neurons exhibited dynamic changes in percentage phasic neurons across blocks (Fig. $2 B$, top). Here, significantly more phasic neurons were observed during block 1 compared with later blocks $\left(\chi^{2}=\right.$ $16.34, p=0.0003$ ). In contrast, there was no difference between phasic percentage during free choice cue presentations preceding the small/immediate option $\left(\chi^{2}=0.42, p=0.8106\right.$; Fig. $2 B$, bottom). However, neurons did shift from being exclusively excitatory in the first block to a having significantly higher proportion of inhibitory neurons in later blocks during free choice cue presentations preceding the small/immediate option (Fisher's exact test, $p=0.001$ ). There were no differences in the ratio of excitatory to inhibitory neurons across blocks for the other trial types (all $p>0.05$ ). These results indicate that phasic PrL neurons preferentially encode the best available predicted option in the absence of delay (i.e., preferring a large magnitude versus small magnitude option in block 1). However, once the delay to receipt increases in blocks 2 and 3, the general population of phasic PrL neurons do not differentially track cue presentation.

PrL neurons also demonstrated changes in firing rate following lever press. Of 115 neurons, 86 (74.8\%) exhibited changes following forced choice lever presses and $62(53.9 \%)$ exhibited changes following free choice lever presses. Phasic neurons were classified as press-excitation (Fig. $3 A$, left) or press-inhibition (Fig. $3 A$, right). On forced choice blocks, there was no significant difference between the percentage of phasic neurons during the lever press across blocks for either large/delay or small/immediate trials ( $p>0.05$ for all analyses, data not shown). Likewise, during free choice large/delay blocks, there was no difference in phasic lever press related neurons across blocks $\left(\chi^{2}=3.02, p=\right.$ 0.2209 ; Fig. $3 B$, top). However, during free choice small/immediate trials, there were fewer phasic neurons to the press during block 1 compared with the other blocks $\left(\chi^{2}=8.92, p=0.0115\right.$; Fig. $3 B$, bottom). Finally, for both trial types, there were no differences in the ratio of excitatory to inhibitory neurons across blocks (all $p>0.05$ ). As with the cue-responsive neurons, these results indicate that phasic PrL neurons preferentially encode the best available option in the absence of delay (i.e., preferring a large-magnitude vs small-magnitude option). However, although populations of both cue- and press-responsive neurons demonstrated altered encoding during block 1 , the nature of this encoding was different. There was an increase in cue-responsive neurons encoding the anticipated large reward during block 1 , whereas there was a decrease in press-responsive neurons encoding the small reward during block 1.

\section{Distinct PrL neurons encode information related to discounted choice}

A substantial proportion of PrL neurons exhibited cue-responsive excitations or inhibitions across large/delay versus small/immediate trials. These neurons were classified as follows: (1) "putative large/delay responsive" neurons, exhibiting a phasic response only during the large/delay cue (Fig. 4, top); (2) "putative small/ immediate responsive" neurons, showing a phasic response only during the small/immediate cue (Fig. 4, middle); or (3) "both (small and immediate)" neurons that demonstrated phasic responses during both large/delay and small/immediate cues (Fig. $4 C$, bottom). During free choice trials, when both cue lights were presented, neurons were classified as putative "large/delay" cueresponsive when the rat chose the large/delay option and putative "small/immediate" cue-responsive when the rat chose the small/ immediate option.

The percentage of free choice phasic neuron populations shifted from "large/delay preferring" to "small/immediate pre- 
A



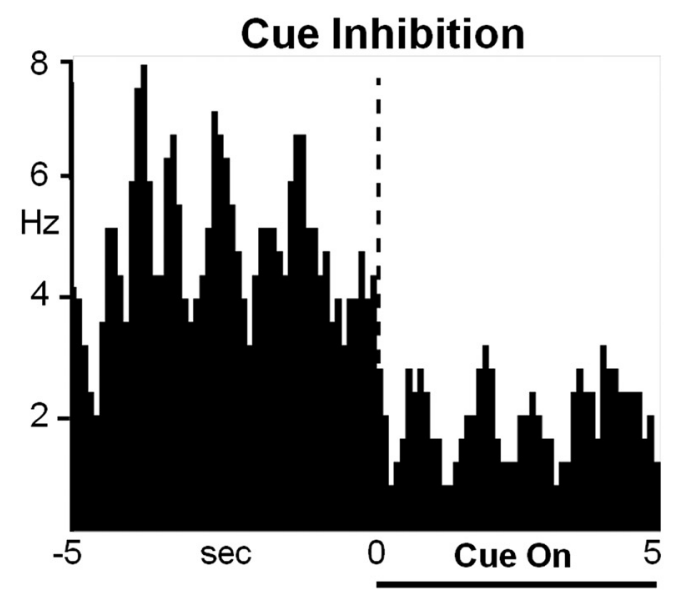

B



Block 2
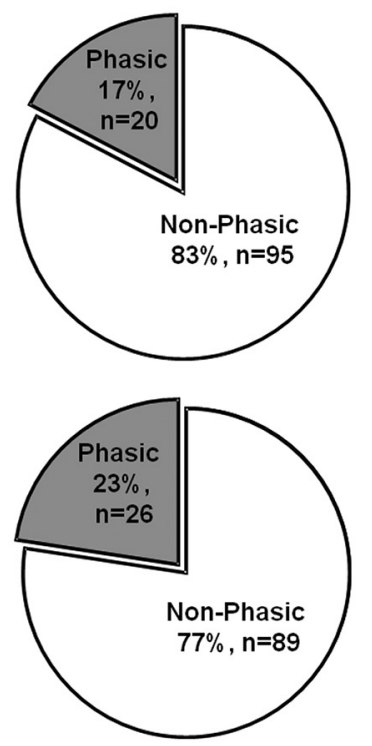

Block 3

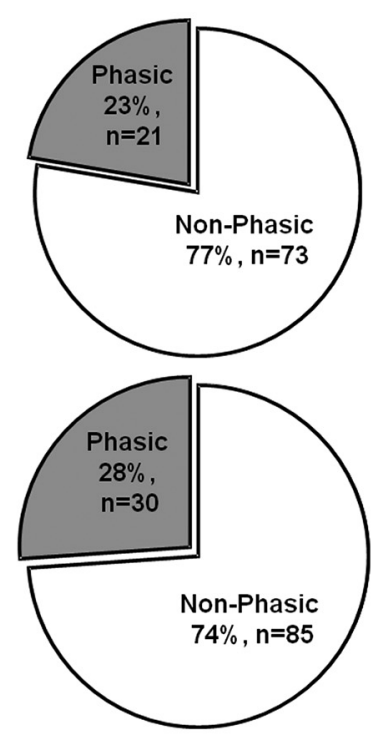

Figure 2. PrL cortical neurons respond to cue presentations. A, Peri-event histograms (PEHs) of representative neurons exhibiting an excitation (left) or an inhibition (right) in firing rate during cue presentations. Data are aligned to cue onset (time 0 , dashed line); cue duration is indicated by horizontal lines below PEHs. B, Pie charts illustrating proportion of neurons that exhibited phasic responses during free choice cue presentations across blocks based upon whether the rat subsequently chose the large/delay reward (top row) or small/immediate reward (bottom row).

ferring" across blocks. This finding is illustrated in Figure $5 \mathrm{~A}$, where each bar graph shows the distribution of each type of putative free choice cue responsive neuron across blocks. Specifically, PrL neurons shifted their population response from mostly "large/delay" to "small/immediate" activity across blocks $\left(\chi^{2}=\right.$ 30.03, $p<0.0001)$. "Small/immediate"-responsive neurons shifted their response profile from exclusively excitatory to partially inhibitory across blocks (block 1, 100\% excitatory; block 2, $100 \%$ excitatory; block 3, 68\% excitatory; Fisher's exact test, $p=$ 0.004 ). This effect was not observed for other trial types (all $p>$ 0.05 ). Interestingly, as shown in Figure $5 B$, putative forced choice phasic responsive neuron populations did not track "preferred" cues across blocks $\left(\chi^{2}=1.48, p=0.83\right)$. Likewise, there were no shifts in the proportion of nonphasic neurons for either trial type (free choice: $\chi^{2}=4.14, p=0.126$; forced choice: $\chi^{2}=1.88, p=$ 0.391; data not shown).

As shown in Figure 5, $C$ and $D$, similar shifts were found for free choice, but not forced choice, putative press-responsive neurons. Specifically, there was a significant decline in large delayresponsive neurons and a corresponding increase in small/ immediate-responsive cells (free choice: $\chi^{2}=31.96, p<0.0001$; forced choice: $\chi^{2}=4.38, p=0.356$ ). Further, differences in the proportion of excitatory: inhibitory press-responsive neurons was observed across conditions. Specifically, putative large/ delay-responsive neurons in forced choice trials following the press significantly shifted from predominantly excitatory to predominantly inhibitory (block 1, 79\% excitatory, block 2, 54\% excitatory; block 3, 39\% excitatory; $\left.\chi^{2}=6.23, p=0.047\right)$. This pattern did not occur during forced choice small/immediateresponsive neurons or free choice trials following the press (all $p>0.05)$. Finally, there were no shifts in the proportion of nonphasic neurons for either trial type (free choice: $\chi^{2}=4.77, p=$ 0.092; forced choice: $\chi^{2}=0.50, p=0.779$ ) or in firing rate across blocks (all $p>0.05)$.

\section{Differences in behavior in HI versus LI rats}

We next separated rats into HI $(n=9)$ and LI $(n=9)$ groups using a median split on average discounting score (the average of delay choice across blocks; Fig. 6A). HI and LI rats significantly differed in delay choice behavior across blocks $\left(F_{(2,32)}=47.27\right.$, 
A

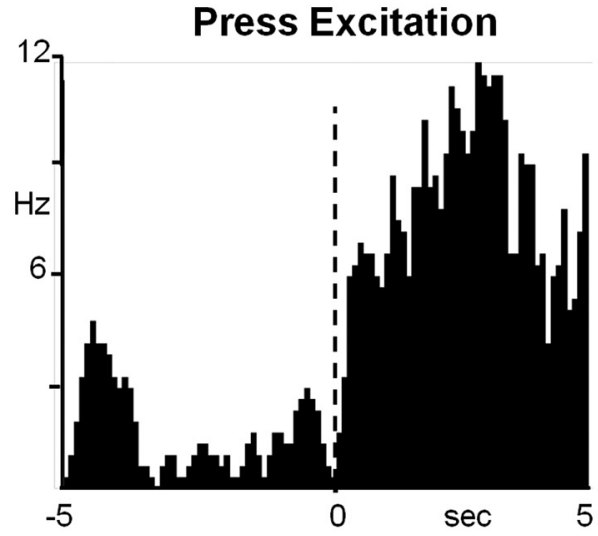

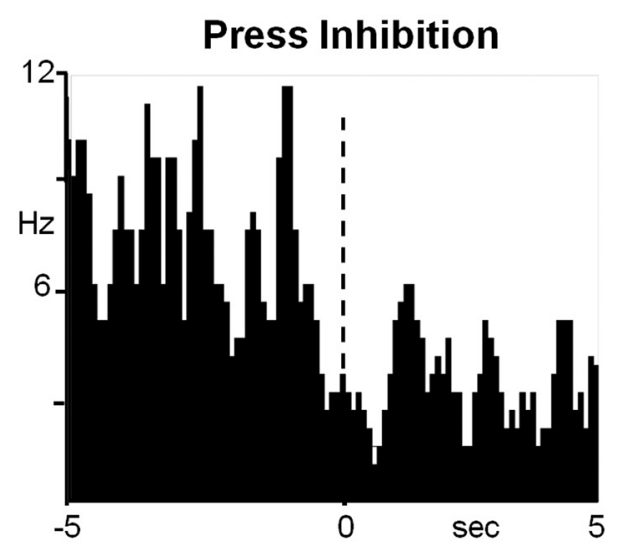

B

Block 1

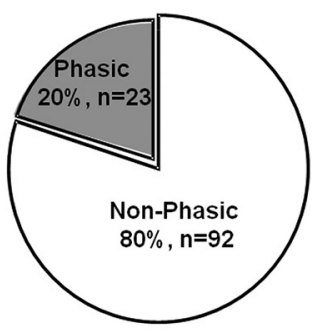

Large/Delay

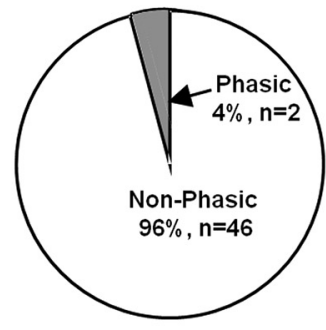

Block 2
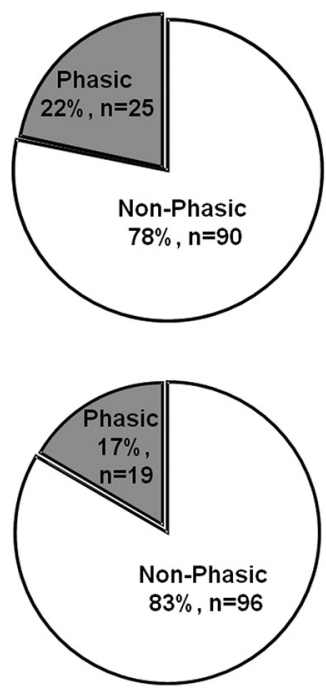

Block 3
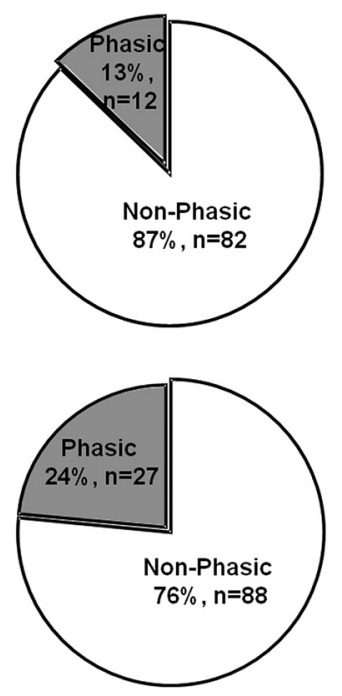

Figure 3. PrL cortical neurons respond to lever press. $A$, Peri-event histograms (PEHs) of representative neurons exhibiting an excitation (left) or an inhibition (right) in firing rate relative to lever press responses. Data are aligned to lever press (time 0 , dashed line). $\boldsymbol{B}$, Pie charts illustrating the proportion of neurons that exhibited phasic responses during free choice lever presses for either the large/delay reward (top row) or the small/immediate reward (bottom row).

$p<0.0001)$ and between impulsivity traits $\left(F_{(1,16)}=59.3, p<\right.$ $0.0001)$, with a significant interaction between impulsivity and block $\left(F_{(2,32)}=6.775, p=0.035\right)$. Both HI rats (Fig. $\left.6 B\right)$ and LI rats (Fig. 6C) were less accurate on block 3 trials $\left(F_{(2,32)}\right.$ $=4.205, p=0.0239)$. However, there was no effect of impulsivity $\left(F_{(1,16)}=1.235, p=0.2828\right)$ or a block $\times$ impulsivity interaction $\left(F_{(2,32)}=0.9905, p=0.3825\right)$ on accuracy. Therefore, impulsivity did not alter accuracy on forced choice trials.

\section{Neuron population dynamics differ between $\mathrm{HI}$ and LI rats}

An important finding of the current study is that the population responses of neurons across cues on free choice trials significantly differed as a function of impulsivity. Population responses for free choice cue-responsive neurons for HI and LI rats are illustrated in Figure 7. Here, cue-responsive neuronal populations in both HI and LI rats shifted from primarily "putative large/delay responsive" to "putative small/immediate responsive" across blocks (HI: $p=0.0013$, Fig. $7 A$; LI: $p=0.0006$, Fig. $7 B$; Fisher's exact test). This indicated that the proportion of cue-responsive neurons reflected all rats' eventual, preferred free choice option. However, whereas there was no significant difference in neu- ron proportions between HI and LI rats in block 1 ( $p=0.071$, Fisher's exact test), there was a difference between HI and LI rats in block $2(p=0.0457$, Fisher's exact test) and block 3 ( $p=$ 0.0037 , Fisher's exact test). These findings indicated that HI rats exhibited significantly more putative "small/immediate" cueresponsive neurons than LI rats in blocks 2 and 3. There were no significant shifts in the PrL's excitatory/inhibitory ratio across blocks for HI or LI rats (all $p>0.05$ ). Likewise, there were no significant shifts in the proportion of nonphasic neurons (all $p>0.05)$.

Population responses for free choice press-responsive neurons for $\mathrm{HI}$ and LI rats are illustrated in Figure 7, $C$ and $D$. Press-responsive neurons exhibited a similar difference between $\mathrm{HI}$ and LI neurons in blocks 2 and 3, but these differences were not statistically significant (block $2: p=0.617$, block $3:=0.124$ ). However, during forced choice trials, LI rats in large/delay responsive neurons shifted from predominantly excitatory to predominantly inhibitory (block 1, 71\% excitatory; block 2, 55\% excitatory; block 3, 21\% excitatory; $\chi^{2}=7.21, p=0.027$ ). Furthermore, in block 3 , this was significantly different from HI rats (Fisher's exact test, $p=0.011$ ). There were no such effects during 
Large/Delay Trials

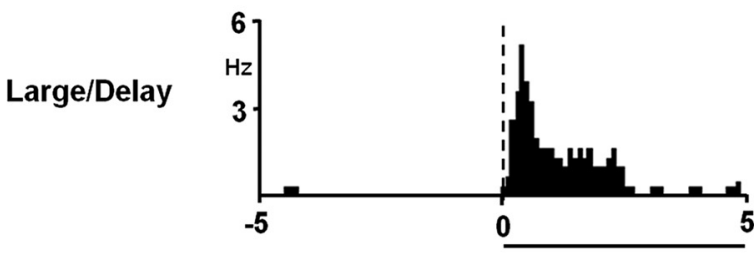

Small/ Immediate
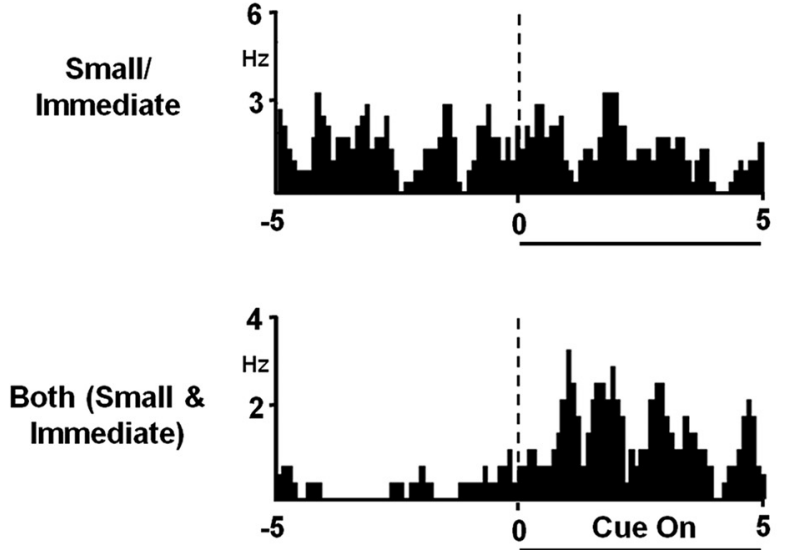

Small/Immediate Trials
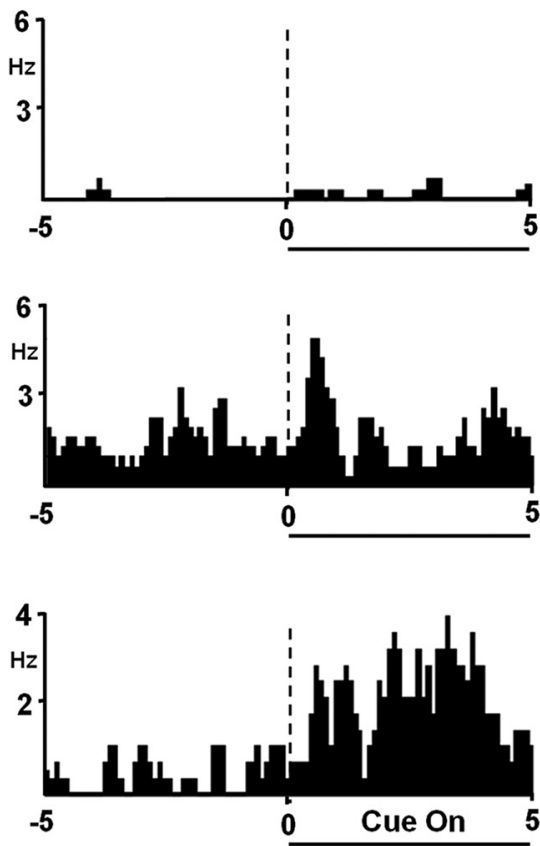

Figure 4. Distinct subsets of PrL neurons selectively track information related to discounted choice. Top, Peri-event histogram (PEH) of a representative neuron exhibiting excitatory firing during cue presentations only during the large/delay (left), but not during small/immediate cue presentations (right). Data are aligned to cue presentation (time 0 , dashed line); duration is indicated by horizontal line below PEHs. Middle, PEH of a representative PrL neuron exhibiting excitatory activity to cues during small/immediate trials (right) not during large/delay cue presentations (left). Bottom, PEHs of a representative neuron that exhibited excitatory activity to both large/delay (left) and small/immediate (right) cue presentations.

A

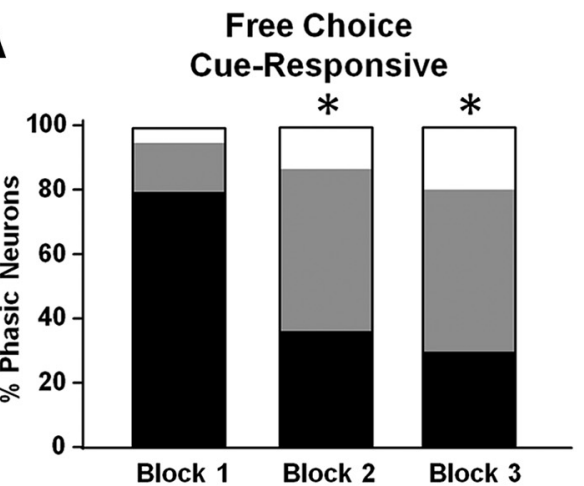

C

Free Choice Press-Responsive

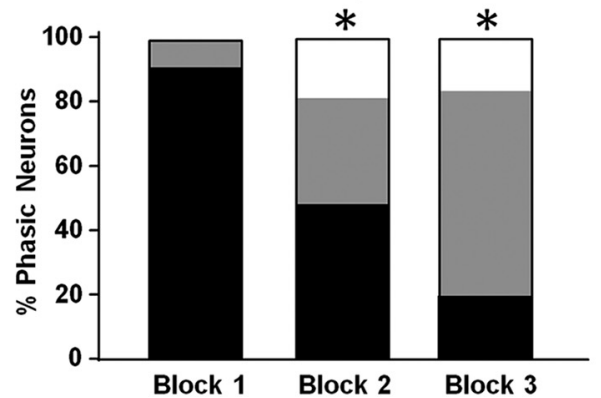

B

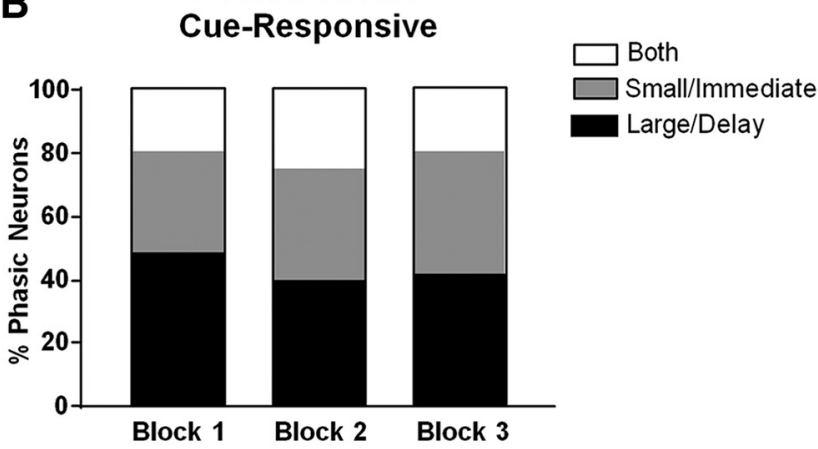

D

Forced Choice

Press-Responsive

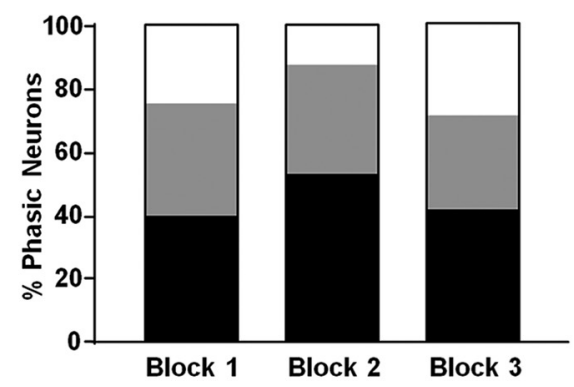

Figure 5. Proportions of event-related (cue, press) responsive neurons dynamically decline across free choice (left) but not forced choice (right) blocks. $\boldsymbol{A}$, Proportion of phasically active responsive neurons to free choice cue presentations. $\boldsymbol{B}$, Proportion of phasically active responsive neurons to forced choice cue presentation. $\boldsymbol{C}$, Proportion of phasically responsive neurons to free choice lever press. $\boldsymbol{D}$, Proportion of phasically responsive neurons to forced choice lever press. ${ }^{*} p<0.05$ compared with block 1. 

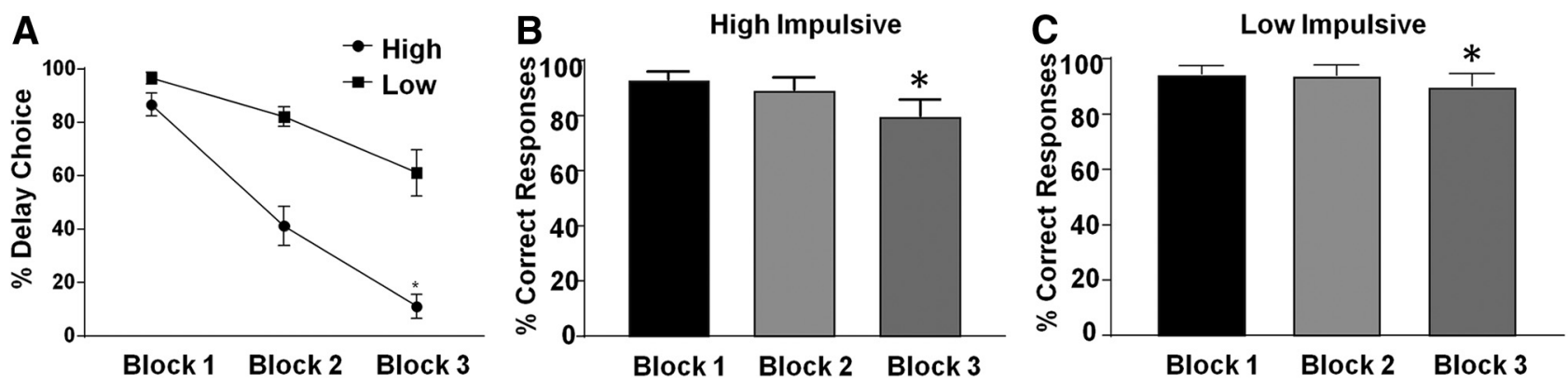

Figure 6. Rats exhibit either high or low levels of impulsivity during the delay discounting task. $\mathrm{HI}, n=3$ males and 6 females; $\mathrm{Ll}, n=6$ males and 3 females. There was no significant difference in sex distribution across groups (Fisher's exact test, $p=0.347$ ). $\boldsymbol{A}$, Percentage delay choice (large reward chosen) during free choice trials in $\mathrm{HI}$ versus LI rats. $\boldsymbol{B}$, Accuracy (percentage correct responses) in $\mathrm{HI}$ rats on recording day. C, Accuracy (percentage correct responses) in LI rats on recording day. ${ }^{*} p<0.05$ compared with block 1.
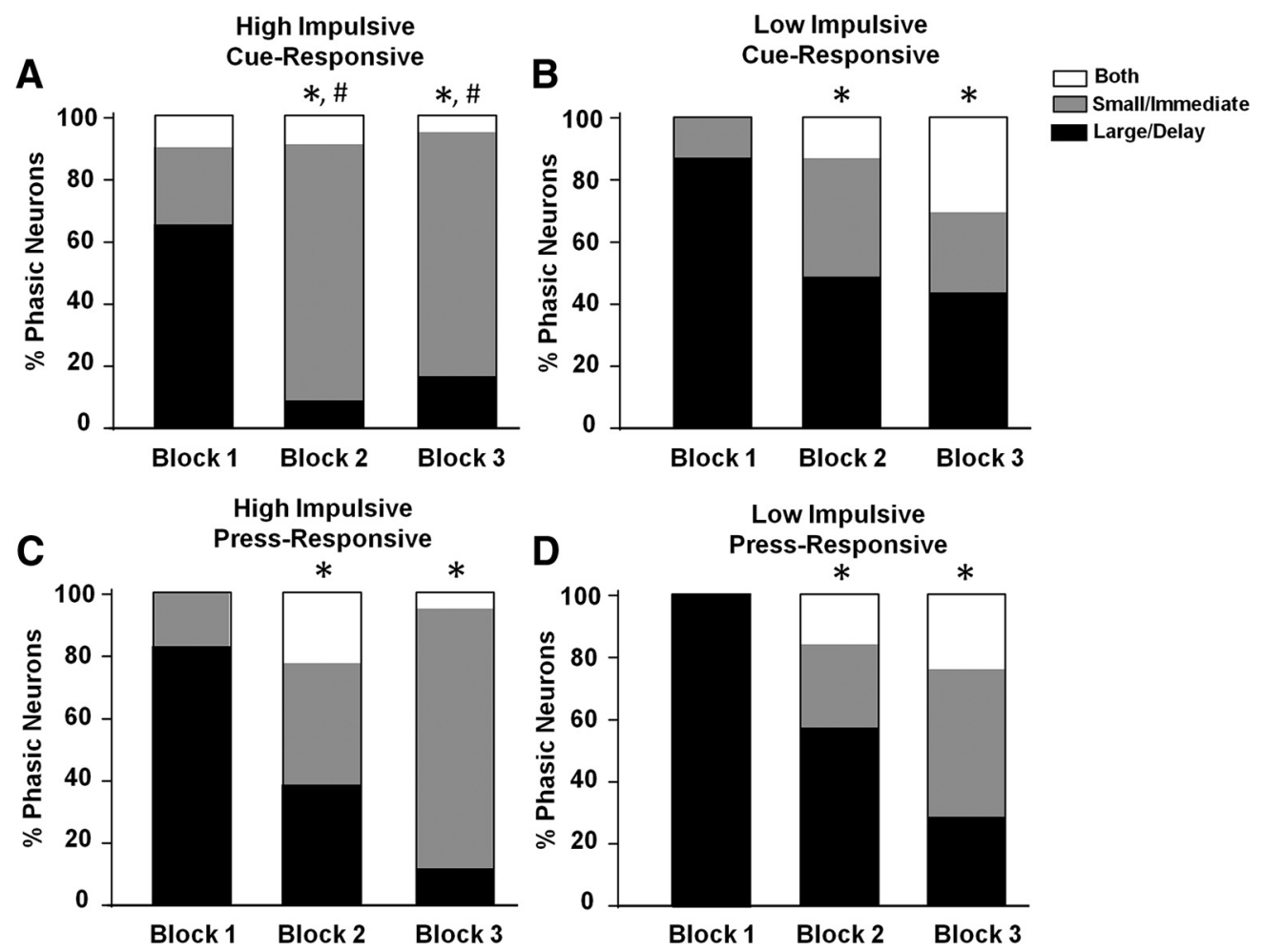

Figure 7. Proportion of neurons exhibiting responsive cell firing during free choice cue presentations in $\mathrm{HI}(\boldsymbol{A})$ versus $\mathrm{LI}(\boldsymbol{B})$ rats across blocks and proportion of neurons exhibiting responsive cell firing during free choice lever presses in $\mathrm{HI}(\boldsymbol{C})$ versus $\mathrm{LI}(\boldsymbol{D})$ rats across blocks. ${ }^{*} p<0.05$ compared with block 1 , \#p<0.05 comparing $\mathrm{HI}$ and $\mathrm{LI}$ rats.

other forced choice trials or during any free choice trials (all $p>$ $0.05)$.

Finally, for all forced choice task events (cue, press), there was no shift in responsive neuron populations across blocks in $\mathrm{HI}$ or LI rats (all $p>0.05$, Fisher's exact test, data not shown). Likewise, for all forced choice task events, there was no difference between $\mathrm{HI}$ and LI rats' responsive neuron populations within any block (all $p>0.05$, Fisher's exact test, data not shown).

\section{Histology}

On test days, 140 neurons were recorded across 130 electrodes. Neurons in the infralimbic cortex $(n=15)$ were excluded from analysis. Of the remaining $125 \mathrm{PrL}$ neurons, waveform analysis revealed that 115 were putative pyramidal (i.e., glutamatergic) neurons and the remaining 10 were excluded from analysis. Tip placements of implanted electrodes positioned in the PrL are shown in Figure 8.

\section{Discussion}

In the present study, PrL cell firing was recorded during discrete elements (cue presentation and lever press) of a delay discounting task. Our findings show that distinct populations of PrL neurons exhibit phasic responses (i.e., increases and/or decreases in activity) to cue presentations or lever press responses during forced choice trials (when rats could respond for only one reward option vs no reward) and free choice trials (when rats could choose between a small immediate vs large delayed reward). However, further analysis revealed that distinct subpopulations of PrL neurons exhibited putative "event-responsive" cell firing wherein they only tracked free choice (but not forced choice) cues across task blocks, reflecting shifts in preferred expected reward value. Conversely, after the lever press, neurons tracked value in both trial types (forced and free choice). This neural encoding varied as a function of inherent level of im- 
pulsivity; HI rats (but not LI rats) showed a greater shift in the proportion of small/immediate cue-responsive neurons as delay to reward increased. Collectively, these findings revealed that unique subsets of PrL neurons track delay discounting behavior and exhibit selective activity that reflects preferred reward value and, importantly, that $\operatorname{PrL}$ dynamic signaling varies as a function of impulsivity level.

PrL neurons track expected value during delay discounting, but only when animals can choose their preferred option

PrL neurons tracked value across different aspects of the delay discounting task; more neurons were responsive (i.e., exhibited excitatory or inhibitory cell firing) following the cue signaling the large, immediate reward, whereas fewer neurons were phasically active following the press for the small, immediate reward. Importantly, in both instances, PrL activity shifted between the 0 and $10 \mathrm{~s}$ delay, but not between the 10 and $20 \mathrm{~s}$ delay. This was in contrast to the animals' behavioral preference, which did shift between the 10 and 20 s delay. This may suggest that the PrL tracks the presence of the delay, but not the changing value of delay, or that the PrL only plays a role in distinguishing delay value at short delays. In support, delay discounting studies that only used short delays $(<20 \mathrm{~s})$ found that lesions/inactivations of the medial prefrontal cortex (mPFC, which includes the PrL) heightened delay discounting (Churchwell et al., 2009; Gill et al., 2010), whereas studies that included short and long delays (between 10 and $60 \mathrm{~s}$ ) found no effect of mPFC manipulation on delay discounting other than a general "flattening" of the discount curve (Cardinal et al., 2001; Feja and Koch, 2014).

In addition to general activity, we also found that distinct PrL neurons were phasic to discrete cue presentations and trial types. That is, unique neuron groups were activated during cue presentations on large/delay, small/immediate, or both trial types. The proportion of these neuronal populations shifted across blocks, from more putative large/delay responsive neurons in the absence of delay (block 1) to more putative small/immediate responsive neurons during the longer delays (blocks 2 and 3). Importantly, this shift occurred only when rats were able to choose their preferred option (free choice trials); when rats only had one option (forced choice trials), PrL neurons did not track expected value. This finding sets the PrL apart from the NAc, BLA, and orbitofrontal cortex, which have all been shown to track expected value of differing magnitudes and delays during both free and forced choice trials (Roesch et al., 2006, 2009, 2010; Saddoris et al., 2015; although see Roesch et al., 2007). Conversely, PrL activity during both free and forced choice trials did track value fol- lowing the lever press. Behavior during free choice trials is likely more deliberative relative to forced choice trials because forced choice trials only require a rat to press the illuminated lever, whereas free choice trials encourage the rat to weigh the distinct outcomes of two possible choices to obtain the subjectively optimal value. Therefore, our findings may suggest that the PrL predominantly tracks expected value in choice situations involving evaluations of the relative costs and benefits associated with different actions.

The present study adds to a growing literature suggesting that value encoding of delay and magnitude is region and context dependent. For example, neurons in the NAc only track cue value if animals make a subsequent choice in that neuron's preferred direction (left or right), suggesting integration between value and action in the NAc (Roesch et al., 2009). Additionally, VTA dopamine neurons only track the highest value during free choice 
trials (as opposed to the value that is eventually chosen, be it high or low), suggesting that these neurons track optimal value rather than expected value in goal-directed contexts (Roesch et al., 2007). Therefore, the PrL may be acting to influence downstream activity in regions such as the BLA or NAc only in those instances when subjective valuation is needed to direct the most appropriate behavior- that is, free choice trials.

\section{Shift in PrL neural activity during delay discounting reflects individual differences in impulsivity}

An interesting finding of the present study was that the shift in PrL activity was dependent on inherent impulsivity. Rats that were more impulsive (e.g., a steeper shift toward the small/immediate option) also showed a greater shift in the proportion of small/immediate neurons across blocks compared with LI rats. This change occurred in cue-activated neurons, indicating that the PrL may contribute to differences in impulsive choice by differentially encoding predicted reward values. Furthermore, during forced choice trials, LI rats showed a stronger shift from excitatory to inhibitory neurons than HI rats following the delay lever press. These findings may suggest that more impulsive individuals have different PrL physiology than LI individuals. In support of this, previous studies reported individual differences in impulsivity that may play a role in the neurobiology of delay discounting. For example, the number of D2 receptors in the PrL is correlated with greater preference for the large/delay reward (Simon et al., 2013). Other brain regions have also reflected differences in impulsivity because the effect of inactivation of the lateral orbitofrontal cortex, anterior insula, and NAc core on delay discounting has been shown to be dependent upon baseline levels of impulsivity (Zeeb et al., 2010; Moschak and Mitchell, 2014; Pattij et al., 2014). Additionally, HI rats exhibited less dopamine release in the NAc core during delay discounting compared with less impulsive rats (Moschak and Carelli, 2017) and in vitro electrical stimulation of NAc elicits less DA release in HI individuals (Diergaarde et al., 2008). However, although many studies have suggested a role for NAc core DA in individual differences in impulsive choice, evidence suggests that DA in the PrL does not signal impulsive choice, but rather availability of reward (delay discounting: Winstanley et al., 2005a; probability discounting: St. Onge et al., 2011). Nonetheless, although DA in the PrL may not influence impulsive choice, the current study demonstrates that HI versus LI rats differ in how PrL neuron populations encode predicted reward value. Therefore, individual differences in PrL physiology may contribute to impulsive choice during delay discounting.

Our current findings may have clinical relevance because the PrL and its human correlate, the ACC, are implicated in disorders associated with heightened delay discounting, such as substance use disorders (Jentsch and Taylor, 1999; Crews and Boettiger, 2009). For example, individuals with substance use disorders demonstrate altered ACC function during delay discounting tasks (Hoffman et al., 2008; Meade et al., 2011). Furthermore, the PrL demonstrates enhanced encoding of cocaine-associated stimuli following abstinence (West et al., 2014), whereas lesions or inactivation of the PrL reduce drug-seeking behavior (McFarland and Kalivas, 2001; Capriles et al., 2003; Di Pietro et al., 2006). Therefore, understanding the neural underpinnings of delay discounting may identify impulsive individuals at risk for psychiatric disorders, or may improve treatments for diseases such as substance use disorders.

\section{Concluding remarks}

The current findings reveal a unique role of PrL neural signaling in discrete aspects of delay discounting. PrL neurons are phasically active to discrete task events, including cue presentation and lever press. These phasic neurons formed unique subgroups that are phasic to the large/delay, small/immediate, or both trial types. These neuron populations tracked the shift in expected reward value as the task progressed, but only when animals were able to make their preferred choice during free choice trials. Further, this tracking was dependent on inherent impulsivity such that HI rats demonstrated a greater shift in putative small/immediateresponsive neurons across blocks. The findings indicate a role for the PrL in encoding impulsive choice as a function of individual impulsivity. Importantly, these findings were similar across male and female rats, suggesting analogous PrL processing during delay discounting in the two sexes. Further investigation of the PrL cortex and its interactions with other brain regions such as the NAc core and BLA is necessary to understand the larger circuit underpinnings of delay discounting and its relationship to impulsivity.

\section{References}

Barkley RA, Edwards G, Laneri M, Fletcher K, Metevia L (2001) Executive functioning, temporal discounting, and sense of time in adolescents with attention deficit hyperactivity disorder (ADHD) and oppositional defiant disorder (ODD). J Abnorm Child Psych 29:541-556.

Capriles N, Rodaros D, Sorge RE, Stewart J (2003) A role for the prefrontal cortex in stress-and cocaine-induced reinstatement of cocaine seeking in rats. Psychopharmacology 168:66-74.

Cardinal RN, Pennicott DR, Sugathapala CL, Robbins TW, Everitt BJ (2001) Impulsive choice induced in rats by lesions of the nucleus accumbens core. Science 292:2499-2501.

Carelli RM, Ijames SG, Crumling AJ (2000) Evidence that separate neural circuits in the nucleus accumbens encode cocaine versus "natural"(water and food) reward. J Neurosci 20:4255-4266.

Churchwell JC, Morris AM, Heurtelou NM, Kesner RP (2009) Interactions between the prefrontal cortex and amygdala during delay discounting and reversal. Behav Neurosci 123:1185-1196.

Crews FT, Boettiger CA (2009) Impulsivity, frontal lobes and risk for addiction. Pharmacol Biochem Behav 93:237-247.

Day JJ, Jones JL, Carelli RM (2011) Nucleus accumbens neurons encode predicted and ongoing reward costs in rats. Eur J Neurosci 33:308 -321.

Devilbiss DM, Spencer RC, Berridge CW (2017) Stress degrades prefrontal cortex neuronal coding of goal-directed behavior. Cereb Cortex 27:29702983.

Diergaarde L, Pattij T, Poortvliet I, Hogenboom F, de Vries W, Schoffelmeer AN, De Vries TJ (2008) Impulsive choice and impulsive action predict vulnerability to distinct stages of nicotine seeking in rats. Biol Psychiatry 63:301-308

Di Pietro NC, Black YD, Kantak KM (2006) Context-dependent prefrontal cortex regulation of cocaine self-administration and reinstatement behaviors in rats. Eur J Neurosci 24:3285-3298.

Evenden JL, Ryan CN (1996) The pharmacology of impulsive behaviour in rats: the effects of drugs on response choice with varying delays of reinforcement. Psychopharmacology 128:161-170.

Feja M, Koch M (2014) Ventral medial prefrontal cortex inactivation impairs impulse control but does not affect delay-discounting in rats. Behav Brain Res 264:230-239.

Gill TM, Castaneda PJ, Janak PH (2010) Dissociable roles of the medial prefrontal cortex and nucleus accumbens core in goal-directed actions for differential reward magnitude. Cereb Cortex. 20:2884-2899.

Heilbronner SR, Rodriguez-Romaguera J, Quirk GJ, Groenewegen HJ, Haber SN (2016) Circuit-based corticostriatal homologies between rat and primate. Biol Psychiatry 80:509-521.

Hoffman WF, Schwartz DL, Huckans MS, McFarland BH, Meiri G, Stevens AA, Mitchell SH (2008) Cortical activation during delay discounting in abstinent methamphetamine dependent individuals. Psychopharmacology 201:183-193.

Jentsch JD, Taylor JR (1999) Impulsivity resulting from frontostriatal dys- 
function in drug abuse: implications for the control of behavior by rewardrelated stimuli. Psychopharmacology 146:373-390.

McClure SM, Laibson DI, Loewenstein G, Cohen JD (2004) Separate neural systems value immediate and delayed monetary rewards. Science 306:503-507.

McFarland K, Kalivas PW (2001) The circuitry mediating cocaine-induced reinstatement of drug-seeking behavior. J Neurosci 21:8655-8663.

Meade CS, Lowen SB, MacLean RR, Key MD, Lukas SE (2011) fMRI brain activation during a delay discounting task in HIV-positive adults with and without cocaine dependence. Psychiatry Res 192:167-175.

Mobini S, Body S, Ho MY, Bradshaw CM, Szabadi E, Deakin JF, Anderson IM (2002) Effects of lesions of the orbitofrontal cortex on sensitivity to delayed and probabilistic reinforcement. Psychopharmacology 160:290298.

Monterosso JR, Ainslie G, Xu J, Cordova X, Domier CP, London ED (2007) Frontoparietal cortical activity of methamphetamine-dependent and comparison subjects performing a delay discounting task. Hum Brain Mapp 28:383-393.

Moorman DE, Aston-Jones G (2015) Prefrontal neurons encode contextbased response execution and inhibition in reward seeking and extinction. Proc Natl Acad Sci U S A 112:9472-9477.

Moschak TM, Carelli RM (2017) Impulsive rats exhibit blunted dopamine release dynamics during a delay discounting task independent of cocaine history. ENeuro 4:ENEURO-0119-17.2017.

Moschak TM, Mitchell SH (2014) Partial inactivation of nucleus accumbens core decreases delay discounting in rats without affecting sensitivity to delay or magnitude. Behav Brain Res 268:159-168.

Moschak TM, Wang X, Carelli RM (2018) A neuronal ensemble in the rostral agranular insula tracks cocaine-induced devaluation of natural reward and predicts cocaine seeking. J Neurosci 1195-1198.

Pattij T, Schetters D, Schoffelmeer AN (2014) Dopaminergic modulation of impulsive decision making in the rat insular cortex. Behav Brain Res 270:118-124.

Paxinos GA, Watson C (1998) The rat brain atlas in stereotaxic coordinates. San Diego: Academic.

Peters J, Büchel C (2010) Episodic future thinking reduces reward delay discounting through an enhancement of prefrontal-mediotemporal interactions. Neuron 66:138-148.

Roesch MR, Bryden DW (2011) Impact of size and delay on neural activity in the rat limbic corticostriatal system. Front Neurosci 5:130.

Roesch MR, Taylor AR, Schoenbaum G (2006) Encoding of timediscounted rewards in orbitofrontal cortex is independent of value representation. Neuron 51:509-520.

Roesch MR, Calu DJ, Schoenbaum G (2007) Dopamine neurons encode the better option in rats deciding between differently delayed or sized rewards. Nat Neurosci 10:1615-1624.
Roesch MR, Singh T, Brown PL, Mullins SE, Schoenbaum G (2009) Ventral striatal neurons encode the value of the chosen action in rats deciding between differently delayed or sized rewards. J Neurosci 29:13365-13376.

Roesch MR, Calu DJ, Esber GR, Schoenbaum G (2010) Neural correlates of variations in event processing during learning in basolateral amygdala. J Neurosci 30:2464-2471.

Saddoris MP, Stamatakis A, Carelli RM (2011) Neural correlates of Pavlovian-to-instrumental transfer in the nucleus accumbens shell are selectively potentiated following cocaine self-administration. Eur J Neurosci 33:2274-2287.

Saddoris MP, Sugam JA, Stuber GD, Witten IB, Deisseroth K, Carelli RM (2015) Mesolimbic dopamine dynamically tracks, and is causally linked to, discrete aspects of value-based decision making. Biol Psychiatry 77:903-911

Simon NW, Beas BS, Montgomery KS, Haberman RP, Bizon JL, Setlow B (2013) Prefrontal cortical-striatal dopamine receptor m RNA expression predicts distinct forms of impulsivity. Eur J Neurosci 37:1779-1788.

St Onge JR, Abhari H, Floresco SB (2011) Dissociable contributions by prefrontal D1 and D2 receptors to risk-based decision making. J Neurosci 31:8625-8633.

Tedford SE, Persons AL, Napier TC (2015) Dopaminergic lesions of the dorsolateral striatum in rats increase delay discounting in an impulsive choice task. PLoS One 10:e0122063.

West EA, Saddoris MP, Kerfoot EC, Carelli RM (2014) Prelimbic and infralimbic cortical regions differentially encode cocaine-associated stimuli and cocaine-seeking before and following abstinence. Eur J Neurosci 39:1891-1902.

Winstanley CA, Theobald DE, Cardinal RN, Robbins TW (2004) Contrasting roles of basolateral amygdala and orbitofrontal cortex in impulsive choice. J Neurosci 24:4718-4722.

Winstanley CA, Theobald DE, Dalley JW, Cardinal RN, Robbins TW (2005a) Double dissociation between serotonergic and dopaminergic modulation of medial prefrontal and orbitofrontal cortex during a test of impulsive choice. Cereb Cortex 16:106-114.

Winstanley CA, Theobald DE, Dalley JW, Robbins TW (2005b) Interactions between serotonin and dopamine in the control of impulsive choice in rats: therapeutic implications for impulse control disorders. Neuropsychopharmacology 30:669-682.

Zeeb FD, Floresco SB, Winstanley CA (2010) Contributions of the orbitofrontal cortex to impulsive choice: interactions with basal levels of impulsivity, dopamine signalling, and reward-related cues. Psychopharmacology 211:87-98.

Zeeb FD, Soko AD, Ji X, Fletcher PJ (2016) Low impulsive action, but not impulsive choice, predicts greater conditioned reinforcer salience and augmented nucleus accumbens dopamine release. Neuropsychopharmacology 41:2091-2100. 